\title{
Sociocultural Determinants to Adoption of Safe Water, Sanitation, and Hygiene Practices in Nyakach, Kisumu County, Kenya: A Descriptive Qualitative Study
}

\author{
Job Wasonga, Mark Okowa, and Felix Kioli \\ School of Arts and Social Sciences, Department of Sociology and Anthropology, Maseno University, Private Bag, Maseno, Kenya \\ Correspondence should be addressed to Job Wasonga; jwasonga@hotmail.com
}

Received 24 June 2016; Revised 11 October 2016; Accepted 17 November 2016

Academic Editor: Santos Alonso

Copyright (c) 2016 Job Wasonga et al. This is an open access article distributed under the Creative Commons Attribution License, which permits unrestricted use, distribution, and reproduction in any medium, provided the original work is properly cited.

Provision of safe water, adequate sanitation, and hygiene has been lauded as one way of preventing diarrheal infections and improving health especially in developing countries. However, lack of safe water, inadequate sanitation, and poor hygiene practices in most parts of rural Kenya have posed a challenge that exposes the populace to diarrhea cases and possible deaths. In this regard, many nongovernmental organizations and governmental agencies have tried to provide water, sanitation, and hygiene services with poor results. This study was conducted using qualitative research methods in Central Nyakach in Kisumu County, Kenya. The methods were focus group discussions (FGD), key informant interviews (KII), and observation of homesteads. The data were then analyzed thematically. Findings revealed that water issues are gendered and its use is socially and culturally categorized. Water storage is affected by traditions such as use of a clay pot, while sanitation and hygiene issues are ritualized and bound by taboos. Latrines are majorly constructed by men and sharing the same with in-laws and older children is prohibited. Children faeces are thrown out in the open fields as a means of disposal and hand washing with soap is nonexistent, since it is believed that doing so would make a person lose the ability to rear livestock. The implications of these findings are that some of these sociocultural practices have a profound effect on health of the population. This affects health care delivery through high incidence rates of disease, encourages "unhealthy" environments through open defecation and pollution, and negates the government's commitment to national and international policies on universal health care provision.

\section{Introduction}

The provision of safe water, adequate sanitation, and hygiene has been lauded as one way of preventing diarrhoeal infections and improving health especially in developing countries. Despite significant investments over the past decades and many unserved people gaining new access to improved water supply and sanitation infrastructure, access to clean and safe water, acceptable sanitation, and effective hygiene remains a persistent challenge with devastating consequences for individuals, economies, and the environment [1]. Research has shown that as many as 30 to 50 percent of water, sanitation, and hygiene (WASH) projects fail after two to five years due to sustainability issues as a result of poor water governance and cultural issues [1]. WASH governance is the broad range of political, social, environmental, economic, and administrative systems that are in place to develop and manage water resources, sanitation, and hygiene services provision at different levels of the society. Governance can also be taken to mean the successful management of community affairs through a mixing of public, private, and voluntary actors, in this case WASH services [2].

A considerable amount of work has been done to identify the benefits of water provision, improving sanitation and hygiene which motivate individual behaviour change among low-income population. Unfortunately, many projects usually provide only one type of service such as improved water source or sanitation with little emphasis on behaviour change component, which develops as a result of perception and attitude acquired over time. Research has also shown that, in spite of efforts to promote behaviour change such as improving washing of hands at key times to prevent 
infection through knowledge and attitude oriented interventions, achievement of such outcomes has been found not to be sustainable [3]. It is generally acknowledged that the introduction of a new concept or technology into a community such as innovation in waste disposal or water supply is not synonymous with its adoption due to many factors. Sociocultural factors have been found to play a major role in the adoption of different attitudes or perception to water and sanitation use as well as hygiene practices.

The benefits of water, sanitation, and hygiene cannot be overemphasized. Indeed, it has been found that provision of safe water, adequate sanitation, and hygiene helps in the reduction of morbidity and mortality of children due to diarrhea, especially those below 5 years old. In spite of the above, the provision of water supply and sanitation alone is not sufficient and must be accompanied by changes in household hygiene behaviour [4-6]. Poor hygiene practices, low sanitation coverage, and reliance on unprotected sources of water often lead to outbreaks of water borne diseases which is very common in Kenya. Kenya has witnessed an upsurge of cholera outbreaks in the past years due to inadequate sanitation and hygiene practices as well as consumption of unsafe water particularly in the Lake Victoria Basin [7]. The poor sanitation in Kenya can partly be attributed to sociocultural issues. It has been estimated that over 43 percent of Kenya's rural population does not have access to basic sanitation and that around 21 million Kenyans use unsanitary or shared latrines [8]. It has also been observed that 5.6 million people practice open defecation (OD), making the country to spend around KES 27 billion (US\$ 324 Million) annually on water, sanitation, and hygiene related problems [9]. Evidence suggests that a modest reduction in diarrhea can be achieved through use of basic improved water and sanitation facilities, while significant reduction can be ensured when water quality is improved from source to point of consumption including hygiene practices such as hand washing [10]. Sociocultural issues hamper most of these positive health practices governing the management and use of water and sanitation facilities. This study, therefore, was undertaken to identify some of the sociocultural determinants to adoption of water, sanitation, and hygiene practices in Nyakach in Kisumu County, Kenya.

\section{Methods}

2.1. Study Setting. This study was conducted in Nyakach central in Kisumu County. Kisumu is one of the 47 counties in Kenya which was created with the adoption of Kenya's new constitutional dispensation in 2010. Nyakach is one of the seven subcounties forming Kisumu County. Nyakach is predominantly inhabited by the Luo who are typically patrilineal and virilocal. The Luo are one of the major ethnic group in Kenya, who consider their entire traditional way of life important and as a resource to be proud of. The social principles regarding age, kinship, and gender are very important in this community and therefore govern their existence and enculturation process.

The Luo of Nyakach depend majorly on subsistence agriculture and fishing with some of them engaging in sand harvesting. Just like in any other part of the Luoland, Luos of Nyakach live in family homesteads, dala, which traditionally comprises a male head of the homestead, his wives or wife and their children, and his married son's families. This setup forms several households within the homestead [11]. The homestead is arranged in a way that the head of the homestead's house, which is usually the first wife's house faces the gate with the sons next to the gate and their doors facing the main house. But with the current economic and other social pressures such as diseases, the homestead arrangement has undergone a significant change in which the female or child being the head of households is becoming common. The arrangement of the homestead is also affected by the introduction of migrant labour which has seen many young and energetic men moving to major towns in search of wage labour.

Nyakach is one of the areas with low water coverage and poor water resource management as well as inadequate sanitation in the county. This contributes to high incidence of diarrheal infections such as cholera. It has also contributed to high incidence of diarrhea and deaths among children aged five years and below which stands at 212/1000 live births. With the devolution of services, there is hope that these trends will be reversed.

2.2. Study Design. This study was part of a larger randomized controlled trial (RCT) which lasted from 2006 to 2012 and was conducted in Kenya to ascertain sustainability of school water, sanitation, and hygiene practices. The study was carried out between May and June 2012, few months before the end of the larger RCT study. This study employed focus group discussions, key informant interviews, and observational activities. There were four focus group discussions lasting between 1 and 2 hours with the first two comprising nine participants, five females and four males, respectively. The third and fourth focus group discussion sessions had ten and eleven participants with female taking six slots, while men taking four and five slots in each of the sessions, respectively. This method allowed the researchers to explore social and cultural beliefs, practices, and norms governing water, sanitation use, and management as well as hygiene. Key informant interviews were conducted with nine opinion leaders and two public health officers. This method was used to obtain individuals' perspectives, including feelings, experience, and opinions on water, sanitation, and hygiene issues. The interviews were done using focus group discussion and key informant interview guides, respectively. An observation tool was used to record information on latrine and bathroom availability, hand washing facilities, presence of dish rack and rubbish pit, and household water treatment chemicals as well as the community water sources.

The discussions were recorded verbatim for both the focus group discussions and key informant interviews using a recorder. Most of the sessions, except only 4 key informant interviews, were conducted in Dholuo, a local language, and translated into English. All research assistants in this study had previous training and experience in qualitative research and professional background in social or health sciences. The focus group discussion sessions were conducted in the nearby schools which were beneficiaries of a school 
health programme $[12,13]$. Some of the limitations of this study are that it used focus group discussion and structural observations which may not be exhaustive in understanding primary cultural practices. In fact, the observation done was majorly focused on sanitation and hygiene facilities presence in the households. These facilities such as presence of latrine and hand washing station can be used as proxy indicators in understanding deeply rooted practices on sanitation and hygiene. Thus, a longer ethnographic study is recommended.

2.3. Ethical Consideration. Verbal informed consent was obtained from the study participants. Confidentiality and anonymity was assured and was maintained throughout the study. Ethical approval was given by the Ethics Review Committee of the Maseno University.

\section{Results and Discussion}

The results of this study have been arranged mainly into two broad categories: use of water and management and sanitation and hygiene practices. These categories were reached after analyzing the contents of the discussions thematically.

3.1. Water Use and Management. The study revealed that water issues are gendered and its use is socially and culturally categorized. Water use is controlled by the person who fetches it which, in most cases, is the female of the household and sometimes accompanied by her children who are usually girls. In a situation where water has to be bought, the man of the household gives the money to buy it while the management remains the work of the woman. This was captured in one of the focus group discussions:

... there are duties for women that they have to do like cooking, collecting water. So even if the man is around he cannot do such because his inner being tells him that it is a woman's duty. Women are the ones who are found in the homes mostly and take care of kitchen matters.

Women have more authority than men because in most cases, men are away fending for the family and women must take charge of the household and kitchen chores. Water belongs to the kitchen which is a domain of women.

Apart from fetching and managing water by women, water storage is affected by traditions such as use of a clay pot. The use of a wide "mouth" traditional clay pot to store drinking water was common and was observed in almost all of the households in the study area. The use of traditional pot with a wide mouth was preferred because it makes the water cool and thus "sweet to drink." This has an impact on the safety of water since women must take charge of water treatment at the household level which, in most of the times, was left to children (the girls) of the house. In this community, just like in many others, water is used for washing, cooking, and sometimes irrigating vegetables in the kitchen gardens and watering animals. This also affects its treatment as was noted by a female key informant that water is treated depending on the kind of work that it should be used for. We only treat water for drinking and not for cooking. Water treatment is a woman's job because when someone, like a visitor comes to drink water, then it is the woman to give it out. It is also expected that women should pass the water treatment skills to their children especially girls. (Adhiambo, 48 years old, Female, House wife and resident of Agoro sub-location).

...men do not have time to go to the kitchen to treat water for household use. It is the girls and women to do that.

There are rituals associated with water use beyond ordinary use of just cooking, washing, and drinking. In this culture, traditionally, water was used for various purposes including religious purposes. Water was used to purify a person when they are facing bad luck or perceived bad omen. It could be mixed with herbs and the person is given to drink no matter the source (of water), whether safe or not. Women with young children could also carry water to their homes of origin when going back for visitation to avoid allergic reactions or other bad omen from befalling their children. This was reported by a female key informant who said that

water is used for various rituals... let's say am living in Nyakach and I want to go to my maternal home in Ugenya with my child, there is a belief that if I go there then the child will be allergic to water. So I carry water from my husband's place to mix with water from the other place [my birth place-Ugenya]. The water is then used for drinking and washing of the child so that the child cannot get sick. (Achieng, 53 years old, Female, grocery trader and vendor)

Everything comes from God including water... and what God has created cannot harm human beings. (John, 55 years old, Male, and Small scale farmer)

although water has been blessed and some people view it as such, population growth and pollution has made most of the community water sources to be unsafe for consumption. We need to treat our water since rivers have been polluted and we cannot be sure about the sources we use whether they are safe or not.

3.2. Sanitation and Hygiene Practices. Sanitation is the provision of adequate excreta or waste disposal facilities that effectively prevent human, animal, and insects contact with the waste [16]. Latrines and rubbish or garbage pits have been found to allow for the safe disposal of waste such as excreta and thus reduce the transmission of diseases. Although the presence of pit latrines was observed in most of the homesteads, its use cannot be guaranteed since it is steeped in beliefs and traditions. In the Nyakach community, pit latrines are majorly constructed by men and sharing the same with 
in-laws and older children is prohibited while children faeces are thrown out in the open fields as a means of disposal. The norms governing latrine use and beliefs surrounding hygiene practices are observed and any deviation from the norm is considered a taboo and attracts chira, a curse. This was captured by a male key informant as follows:

A latrine that is used by the mother or father inlaw is not to be used by the daughters or sons. It is common to observe two latrines, in the upper side and the lower side of homesteads, especially for those who are capable of constructing latrine. It was a rule for the sons who have built their own houses within the homestead to have their own latrines. (Ochido, 38 years old, Male, a peasant farmer and fisherman)

latrines should not be used at night since they harbor evil spirits...so we dig holes behind the houses to defecate.

Latrine use was also found to be affected by cost of construction and poverty or lack of resources as well as the soil condition. The study found out that many of the community members are not able to construct latrines due to the cost involved since the majority of them are poor. According to a male key informant,

it is hard to have latrines since they are destroyed during rainy season. Our soil is weak and requires that we start building the latrine from the deep down the hole. If you walk in the community you will find that some homes do not have latrines since they have sunk or spoilt. This makes people to resort to use of the bushes since they are embarrassed to request of their neighbours. (Onditi, 44 years old, Male, Laborer and community leader)

construction of pit latrines in this area is hampered by loose soil that makes it a very expensive venture. Any latrine which is constructed without the pit being lined from down will definitely collapse.

The study also sorts to find out the sociocultural factors which hinder hand washing with soap. It was found that hand washing with soap is nonexistent, since it is believed that doing so will make a person lose the ability to rear livestock. Among the Luo, rearing of livestock is highly regarded and is considered as a sign of wealth. The study participants noted that livestock give a person social status and are a source of pride. Livestock are also used as bride price and, therefore, enable a father to bequeath some animals to a son for marriage purposes. Failure to rear animals or keep them may thus be considered a deviation from norm.

\section{Conclusion}

Results from this study, if considered along other similar studies, may assist water sanitation and hygiene practitioners to come up with sustainable interventions. It is common knowledge that diseases that afflict majority of the population, particularly in developing countries such as Kenya, stem from lack of safe water, poor sanitation, and unhygienic practices. Indeed, this study has shown that water management practices are gendered and that women and children play a major role in household water management. Thus, any intervention on household water management or point of use water quality improvement should target them. Secondly, it has been found that safe water provision, adequate sanitation, and good hygiene practices are paramount for any country to reduce morbidity and mortality associated with diarrhea, especially among children. This study has shown that some practices such as throwing children's faeces in the bushes or not sharing the same latrine with in-laws may encourage open defecation or contaminate the environment or water sources leading to exposure of the population to faecal-oral diseases. Other habits or practices which discourage hand washing with soap may also contribute to spread of diarrhea. In this regard, there is need to formulate policies that will encourage investment in this sector, while considering the sociocultural issues and factors which are very critical for successful implementation of any programme. Policies are important, but their implementation and acceptance by the population that it serves are the only way to improve on some of the practices which may work against good health due to cultural orientation. Although the study was limited in its scope and methodology, there is need for a comprehensive ethnographic study to fully understand hygiene and related practices affecting water management and sanitation in different cultural contexts.

\section{Competing Interests}

The authors declare that they have no competing interest.

\section{Acknowledgments}

This study was done under the umbrella of SWASH+, an applied research funded by Bill and Melinda Gates Foundation and implemented by CARE International in Kenya, Emory University, Great Lakes University of Kisumu and http://water.org/.

\section{References}

[1] UNDP/UNICEF, "WASH and accountability: explaining the concept," in Accountability for Sustainability Partnership, UNDP Water Governance Facility at SIWI and UNICEF, Stockholm, Sweden, 2015.

[2] S. van der Geest and N. Obirih-Opareh, "Getting out of the shit: toilets and the daily failure of governance in Ghana," Bulletin de l'APAD, no. 23-24, 12 pages, 2002.

[3] L. E. Greene, M. C. Freeman, D. Akoko, S. Saboori, C. Moe, and R. Rheingans, "Impact of a school-based hygiene promotion and sanitation intervention on pupil hand contamination in Western Kenya: a cluster randomized trial," American Journal of Tropical Medicine and Hygiene, vol. 87, no. 3, pp. 385-393, 2012. 
[4] A. M. Cairncross, "Health impacts in developing countries: new evidence and new prospects," Journal of the Institution of Water and Environmental Management, vol. 4, no. 6, pp. 571-577, 1990.

[5] T. E. Mertens, S. Jaffar, M. A. Fernando, S. N. Cousens, and G. Feachem, "Excreta disposal behaviour and latrine ownership in relation to the risk of childhood diarrhoea in Sri Lanka," International Journal of Epidemiology, vol. 21, no. 6, pp. 11571164, 1992.

[6] V. Curtis, S. Cairncross, and R. Yonli, "Review: domestic hygiene and diarrhoea-pinpointing the problem," Tropical Medicine \& International Health, vol. 5, no. 1, pp. 22-32, 2000.

[7] D. Olago, M. Marshall, S. O. Wandiga et al., "Climatic, socioeconomic, and health factors affecting human vulnerability to cholera in the Lake Victoria basin, east Africa," Ambio, vol. 36, no. 4, pp. 350-358, 2007.

[8] KDHS, "Kenya demographic and health survey 2008-9," in Kenya Demographic and Health Survey, Statistics KNBo, Ed., Government of Kenya, Nairobi, Kenya, 1998.

[9] WorldBank, Kenya Loses KES27 Billion Annually due to Poor Sanitation. Economic Impacts of Poor Sanitation in Africa, World Bank, Washington, DC, USA, 2012.

[10] World Health Organization, Preventing Diarrhoea Through Better Water, Sanitation and Hygiene: Exposures and Impacts in Low-and Middle-Income Countries, World Health Organization, Geneva, Switzerland, 2014.

[11] E. L. Grigorenko, P. W. Geissler, R. Prince et al., "The organisation of Luo conceptions of intelligence: a study of implicit theories in a Kenyan village," International Journal of Behavioral Development, vol. 25, no. 4, pp. 367-378, 2001.

[12] M. C. Freeman, L. E. Greene, R. Dreibelbis et al., "Assessing the impact of a school-based water treatment, hygiene and sanitation programme on pupil absence in Nyanza Province, Kenya: a cluster-randomized trial," Tropical Medicine \& International Health, vol. 17, no. 3, pp. 380-391, 2012.

[13] J. Wasonga, C. O. Olang'o, and F. Kioli, "Improving Households Knowledge and Attitude on Water, Sanitation, and Hygiene Practices through School Health Programme in Nyakach, Kisumu County in Western Kenya," Journal of Anthropology, vol. 2014, Article ID 958481, 6 pages, 2014.

[14] D. Rajaraman, K. S. Varadharajan, K. Greenland et al., "Implementing effective hygiene promotion: lessons from the process evaluation of an intervention to promote handwashing with soap in rural India," BMC Public Health, vol. 14, article 1179, 2014.

[15] H.-J. Mosler, "A systematic approach to behavior change interventions for the water and sanitation sector in developing countries: a conceptual model, a review, and a guideline," International Journal of Environmental Health Research, vol. 22, no. 5, pp. 431-449, 2012.

[16] J. Wasonga and F. Bukania, "Sanitation and physical disability: challenges to latrine access in Kakuma refugee camp, Kenya," Waterlines, vol. 34, no. 2, pp. 174-185, 2015.

[17] A. Dittmer, "Towards Total Sanitation: Socio-cultural barriers and triggers to total sanitation in West Africa," WaterAid Report $12,2009$. 


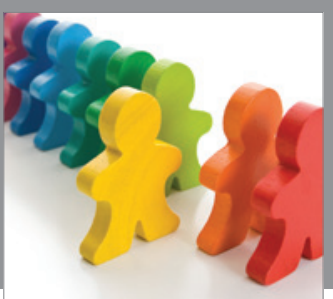

Autism

Research and Treatment
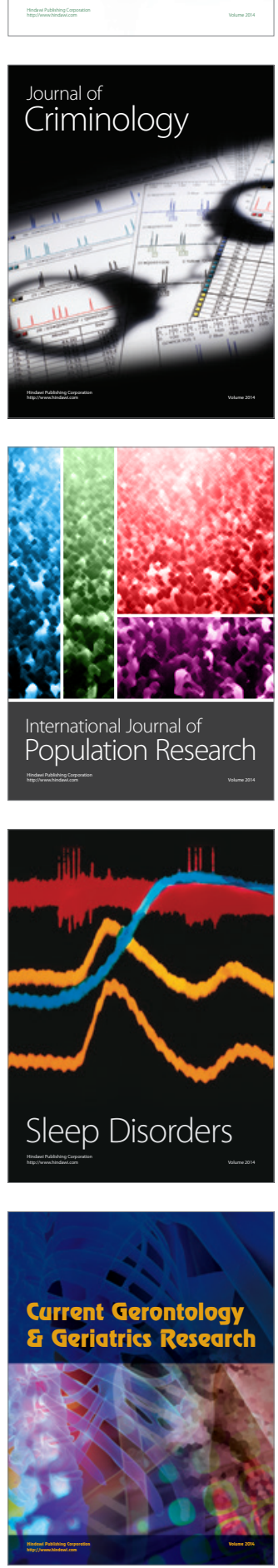

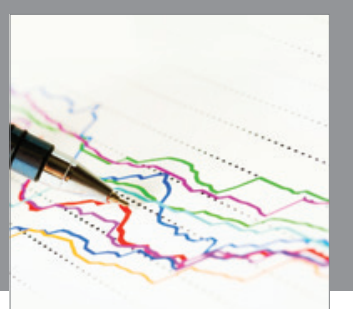

Economics

Research International
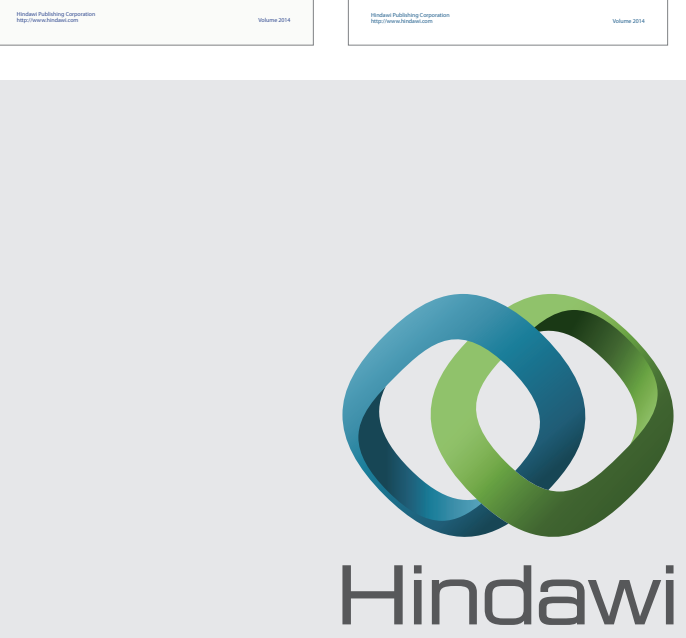

Submit your manuscripts at

http://www.hindawi.com
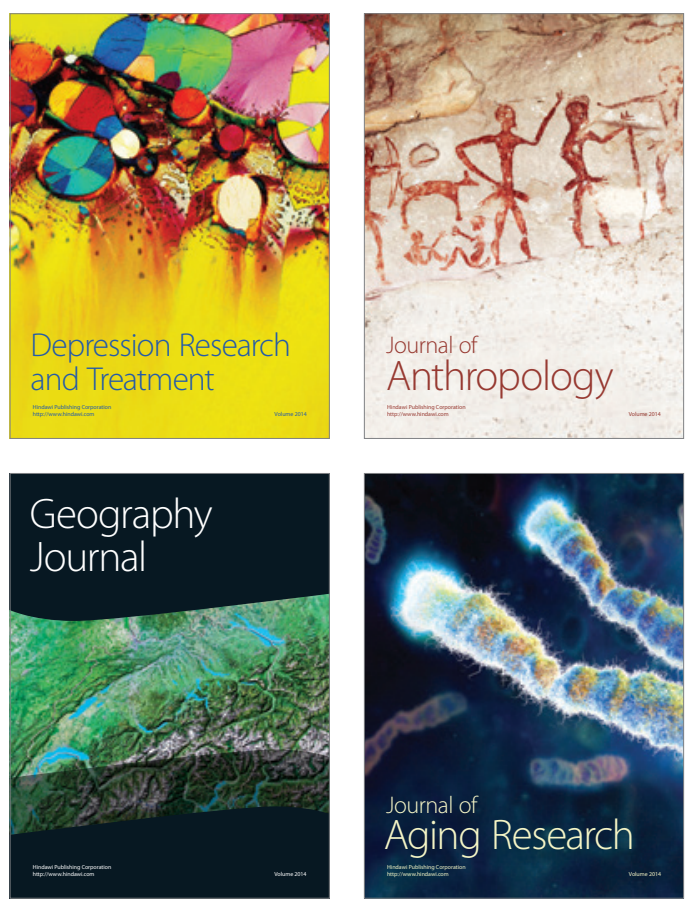
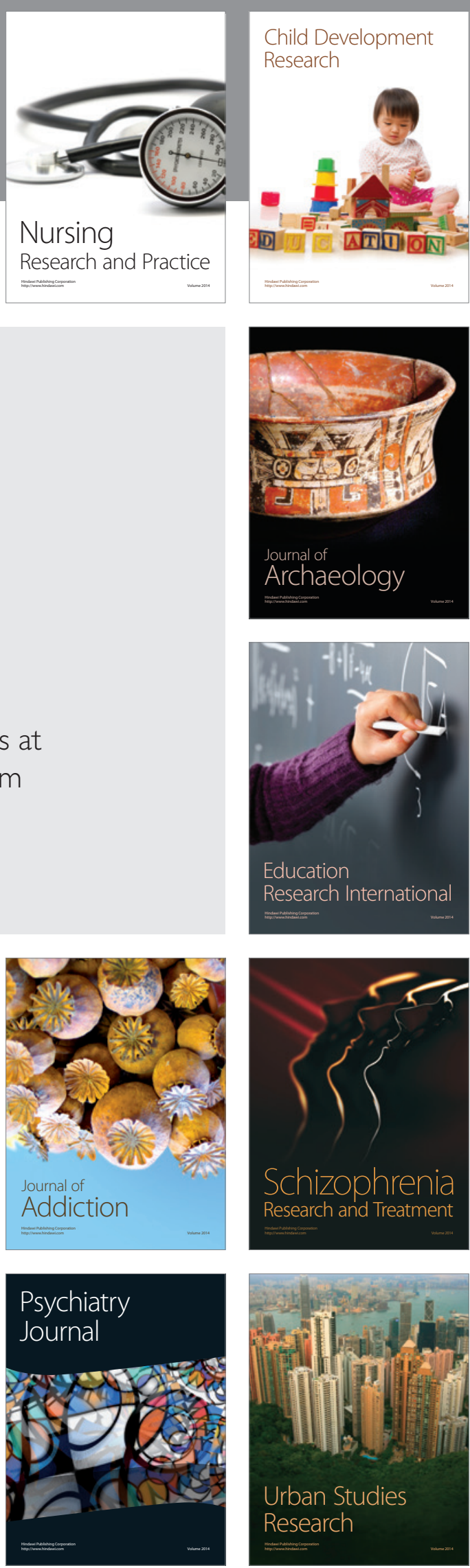